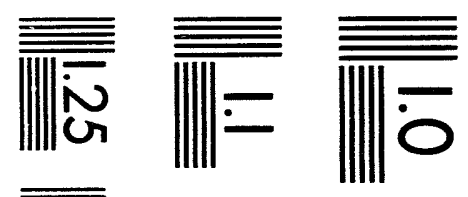

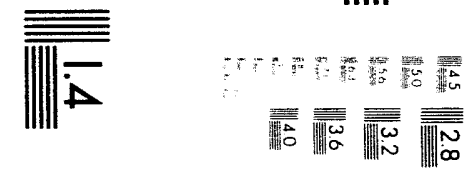

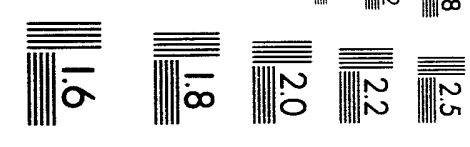


LA-UR- 93-3677

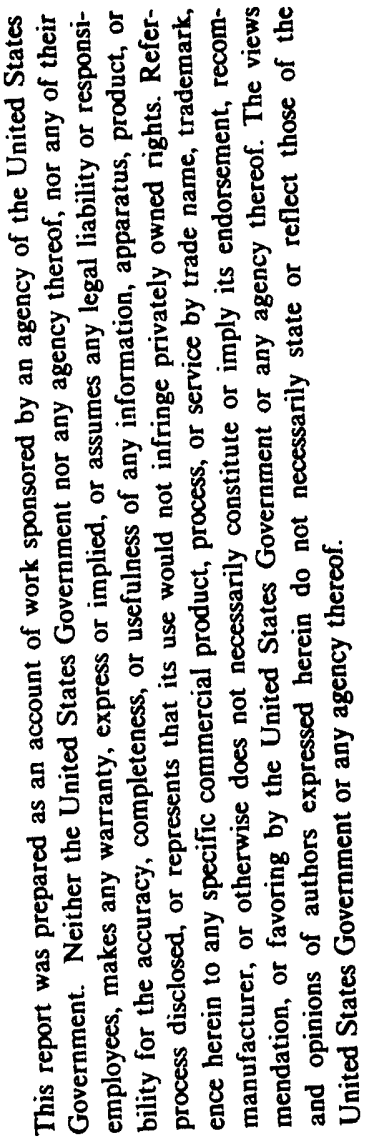

Title:

Author(s):

Submitted to:

Ronald L. Miller

J. D. Galambos

S. W. Haney

I. J. Perkins

J. Mandrekas

Tokamak Physics Experiment (TPX) Cost and Performance Trade Studies Using Supercode

15th IEEE/NPSS Symposium on Fusion Engineering

October 11-15, 1993 Hyannis, MA

MASTER

DISTRIBUTION OF THIS DOCUMENT IS UNLIMITED

nos

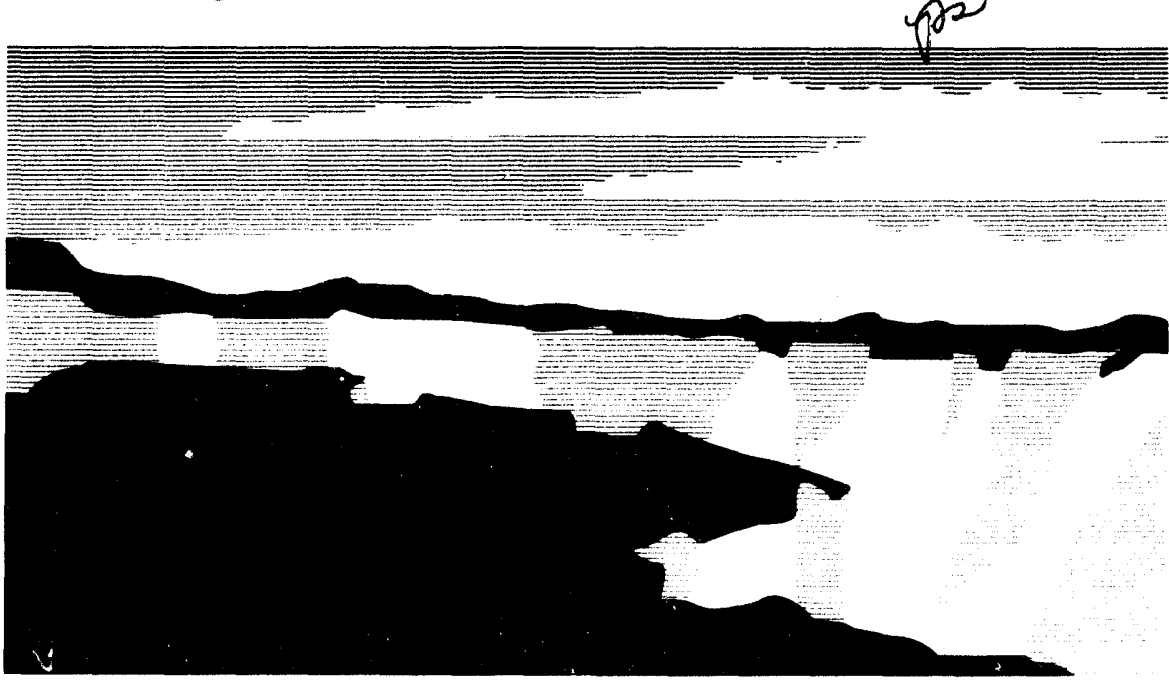

NATIONAL LABORATORY

Los Alamos National Laboratory, an affirmative action/equal opportunity employer, is operated by the University of California for the U.S. Department of Energy under contract W-7405-ENG-36. By acceptance of this article, the publisher recognizes that the U.S. Government retains a nonexclusive, royalty-free license to publish or reproduce the published form of this contribution, or to allow others to do so, for U.S. Government purposes. The Los Alamos National Laboratory requests that the publisher identify this article as work performed under the auspices of the U.S. Department of Energy. 


\title{
Tokamak Physics Experiment (TPX) Cost and Performance Trade Studies Using SUPERCODE ${ }^{\dagger}$
}

\author{
R. L. Miller \\ Los Alamos National Laboratory, P.O. Box 1663, Los Alamos, NM 87545, USA \\ J. D. Galambos \\ Oak Ridge National Laboratory, Oak Ridge, TN 37831, USA \\ S. W. Haney, L. J. Perkins \\ Lawrence Livermore National Laboratory, Livermore, CA 94550, USA \\ J. Mandrekas \\ Georgia Institute of Technology, Atlanta, GA 30332, USA
}

\begin{abstract}
The Tokamak Physics Experiment (TPX) has been proposed to demonstrate steady-state operation and to develop advanced performance in terms of $\beta$ and energy confinement. Major TPX cost drivers and the impact of physics and technology constraints and options on operating scenarios are identified. Key trade and sensitivity studies performed using SUPERCODE are summarized.
\end{abstract}

\section{INTRODUCTION}

The Tokamak Physics Experiment (TPX) is the next major proposed step in the U. S. magnetic fusion program [1-3]. To be sited at the Princeton Plasma Physics Laboratory (PPPL), TPX has the mission $|4|$ to develop the scientific basis for an economic, compact, and steady-state tokamak fusion reactor, requiring the development and demonstration of stable, steady-state tokamak operating regimes with high $\beta$, high confinement, and high boostrap-current fraction, consistent with ARIES recommendations [5]. The TPX engineering design incorporates superconducting magnets, actively cooled divertor and first-wall systems, and in-vessel remote maintenance. The deuterium plasma heating and current-drive systems include neutral beams, ICRH, and lower hybrid.

As part of the TPX conceptual design effort, the SUPERCODE [6], combining physics, engineering, and cost models, has been used to perform trade and optimization studies to establish the context of the reference design. Major TPX cost drivers and the impact of physics and technology constraints and options on operating scenarios are identified. Some results of key trade and sensitivity studies are described below.

†This work was supported hy the U.S. Department of Energy at L.ANL, under (ontract No, W-7405-ENG-36 and ICO No. S-03563-A hetween PIPI, and LANL, at ORNL under Contract No. DE-ACO584OK2140\%, at LLNL under Contract No. W-7405-ENG-48, and at GlT under Contract No. DE-FG0590E52141.

\section{COST MODEL}

The TPX Work Breakdown Structure (WBS) defines and organizes the activities and subsystems which comprise the machine and associated projected costs. The Plant and Capital Equipment (PACE) direct cost is the sum of Hardware, Engineering, Installation, and Contingency costs. Operating Expenses (OPEX) include direct costs for Research and Development, Mockups, Conceptual Design, ctc.. General \& Administrative (G\&A) PACE (9\%) and OPEX (55\%) indirect rates, representative of major US fusion laboratory experience, are applied to the direct costs to determine the total PACE and total OPEX costs, which then sum to yield the estimated Total Project Cost (TPC), about $510 \mathrm{M} \$$ (1993 US dollars). For each specific WBS item and cost category, cognizant engineers have recommended a cost algorithm based on the TPX conceptual design activity. Often the cost algorithms are specific to the PPPL site, because credit has been taken for use of the existing TFTR facilities. The reference cost model [7] has been maintained and updated as required at PPPL, and was the basis for the recent Independent Cost Estimation (ICE) cost review and validation. A derivative model, compatible with SUPERCollk, provides the opportunity to perform trade and sensitivity studies, using cost as an object function or constraint. The details of the SUPERCOLE cost module are described elsewhere $|8|$.

\section{RESULTS}

TPX parameters are taken to be linked by the Troyon relation, $\beta=\beta_{N} I_{p} / a B_{T}$, where $I_{n}$ (MA) is the toroidal plasma current, $B_{T}(\mathrm{~T})$ is the on-axis toroidal magneticfield strength, and $a(\mathrm{~m})$ is the plasma half-width at the midplane. Two distinct operating scenarios follow from the TPX mission: Standard high-beta Tokamak (ST), consistent with the present tokamak data base at $\beta_{N} \simeq$ 0.035 and $q_{\psi} \simeq 3.3$, and the Advanced-performance tokamak (AT), consistent with $\beta_{N} \simeq 0.05-0.07$ and 


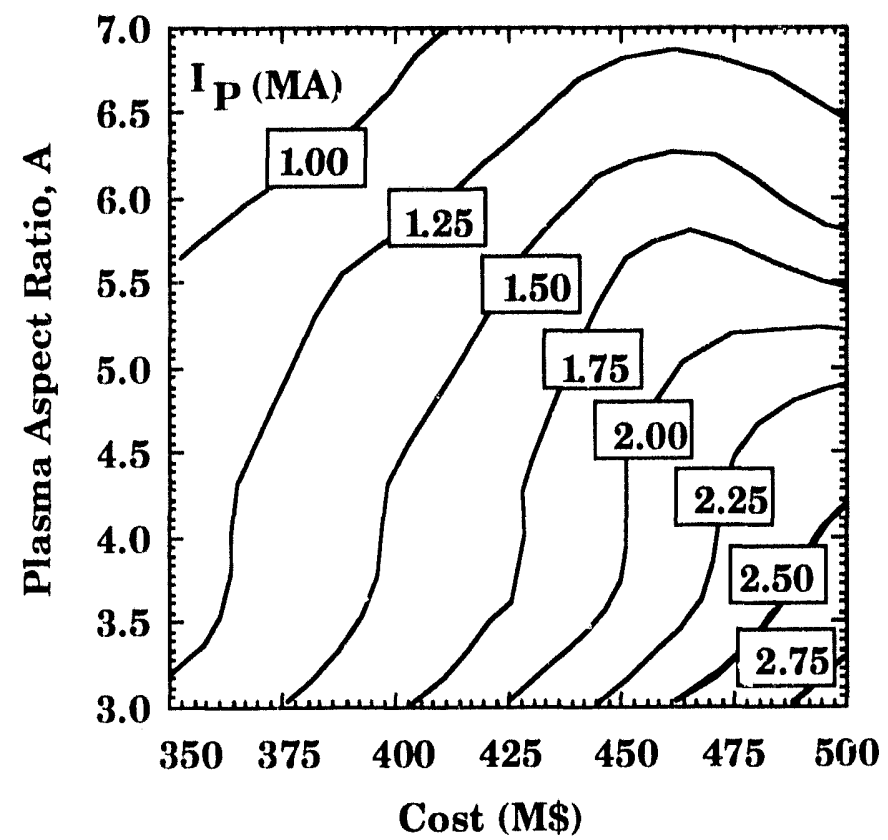

Fig. 1. A-\$ phase space for TIX illustrating contours of constant plasma current, $I_{p}$ (MA), for the ST scenario.

an energy confinement time, $\tau_{E}$, e: hancement factor, $\| \simeq 3-4$, rather than 2 for the ST scenario.

Using SUPERCOLE, key trade and sensitivity studies have been performed. A two-dimensional phase space of plasma aspect ratio, $A=R_{T} / a$, and device cost was selected to display the SUPERCoDE results. In this A-\$ space, contours of selected device parameters (e.g., plasma current, on-axis toroidal magnetic field strength, heating power, etc.) are displayed, as in Figs. 1,2. All design points in this phase space meet the usual constraints of the ST scenario; the density constraints were not applied. At each $[A, \$]$ point the triple product, $n \tau_{E} T$, is maximized. TPX devices with $I_{p} \geq 2.0 \mathrm{MA}$ and $B_{T} \geq 3.0 \mathrm{~T}$ imply $1992-\$$ device costs greater than $450 \mathrm{M} \$$ and plasma aspect ratios in the range, $A=4-5$. For fixed $A$, device cost is relatively insensitive to $B_{T}$, unless $B_{T} \geq 3.50 \mathrm{~T}$. Lower cost devices with reduced ST and AT performance are of marginal interest.

The capability for bi-modal optimization studies was added to SUPERCODE to allow the identification of TPX devices that meet the distinct requirements of both ST and AT scenarios. Modal variables/constraints (e.g., $n$, $\left.T, I_{P}\right)$ are duplicated in each mode and treated separately. Synchronized variables (e.g., $R_{T}$, TF-coil build) have the same value in all modes and are governed by synchronized constraints. Composite variables (i.e., the largest, encompassing particular modal variables) are subject to mode-specific constraints. The multitasking capabilities of UNIX are invoked to clone copies of SUI'ERC(OIE to compute modal quantities.

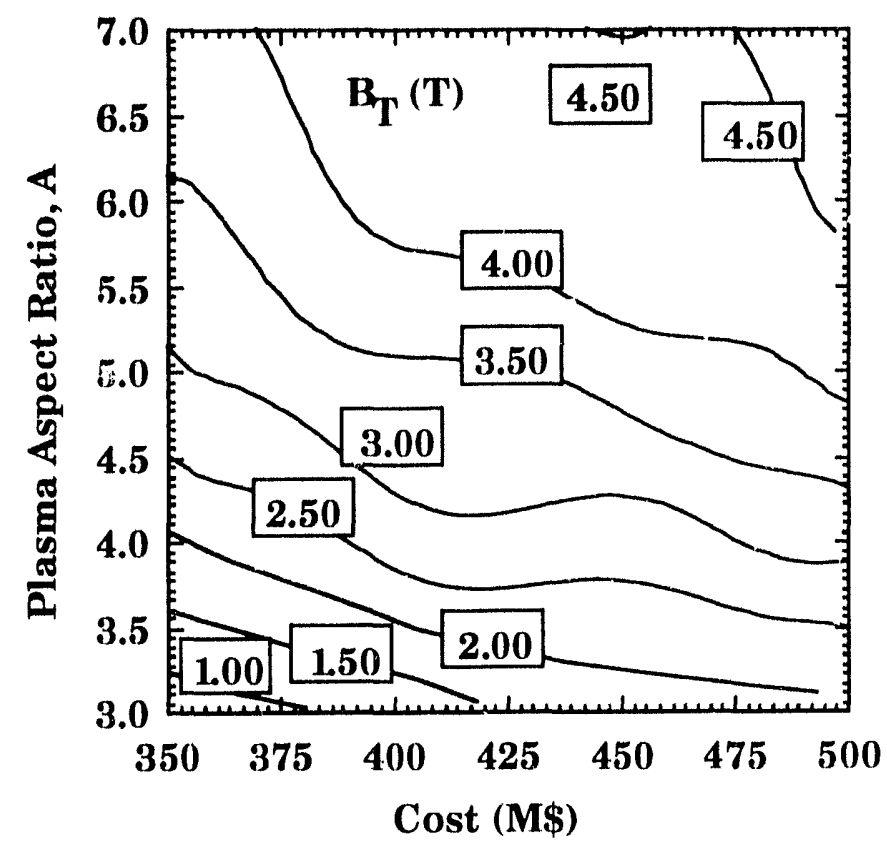

Fig. 2. A-\$ phase space for 'I'P illustrating contours of constant magnetic-field strength, $B_{T} T$ ('T), for the S'T' scenario.

The results of Fig. 3 are used to address the question: What is the least expensive machine that satisfies both the ST and AT missions? The on-axis toroidal magnetic field, $B_{T}$, is either fixed (synchronized) or varied, as indicated. Either the Day-1 or upgraded Day-n heating capabilities are invoked, as shown. The Day-n upgrade assumes $24 \mathrm{MW}$ of both NBI and ICRH power. Device cost shows a minimum near $A \simeq 4.5-5$ under all indicated assumptions. The miniritum size device meeting both ST and AT requirements, occurs with plasma aspect ratio, $A \simeq 4.5$, as indicated by Fig. 4. Here, the plasma geometric parameters are insensitive to whether the on-axis toroidal magnetic field, $B_{T}$, is treated as a fixed (synchronized) variable or not.

The required external heating powers depend on the value of plasma aspect ratio, $A=R_{T} / 1$, and the choice of ST virsus AT operating mode. The neutral-beam heating power as a function of plasma aspect ratio is shown in Fig. 5; the curves for AT mode (denoted by squares and triangles) are nearly coincident. Fig. 6 shows ICRH heating power under the same conditions. Total auxiliary heating power is minimized for $A \simeq 4.5-5$. The Day-1 constraints on heating power preclude high-current, low-A devices.

These sample SuperColle results point to the selection of TPX parameters that maximize performance in both ST and AT operating modes while minimizing device cost. Major parameters of the TPX conceptual design are summarized in Table I. 


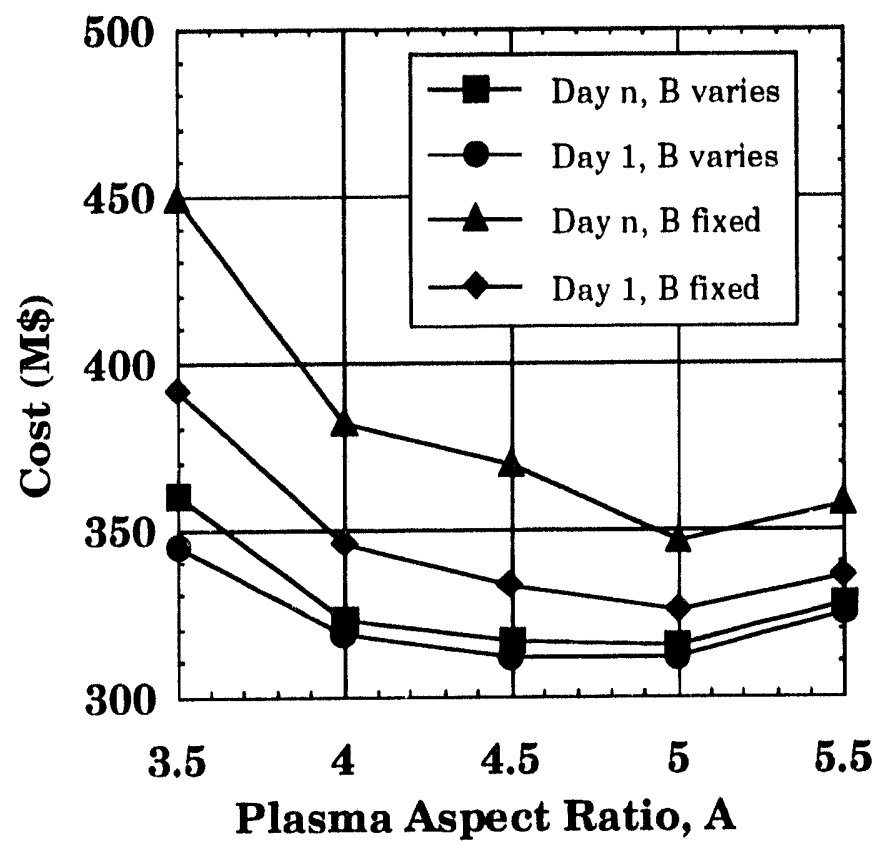

Fig. 3. TPX device cost $(\mathrm{M} \$)$ as a function of plasma aspect ratio, $A=R_{T} / a$, assuming fixed (synchronized) or varied on-axis magnetic field, $B_{T}$, and either Day-1 (NBI: $8 \mathrm{MW}$, ICRH: 8 $\mathrm{MW}$ ) or Day-n (NBI: $24 \mathrm{MW}, \mathrm{ICRH}: 24 \mathrm{MW}$ ) heating.

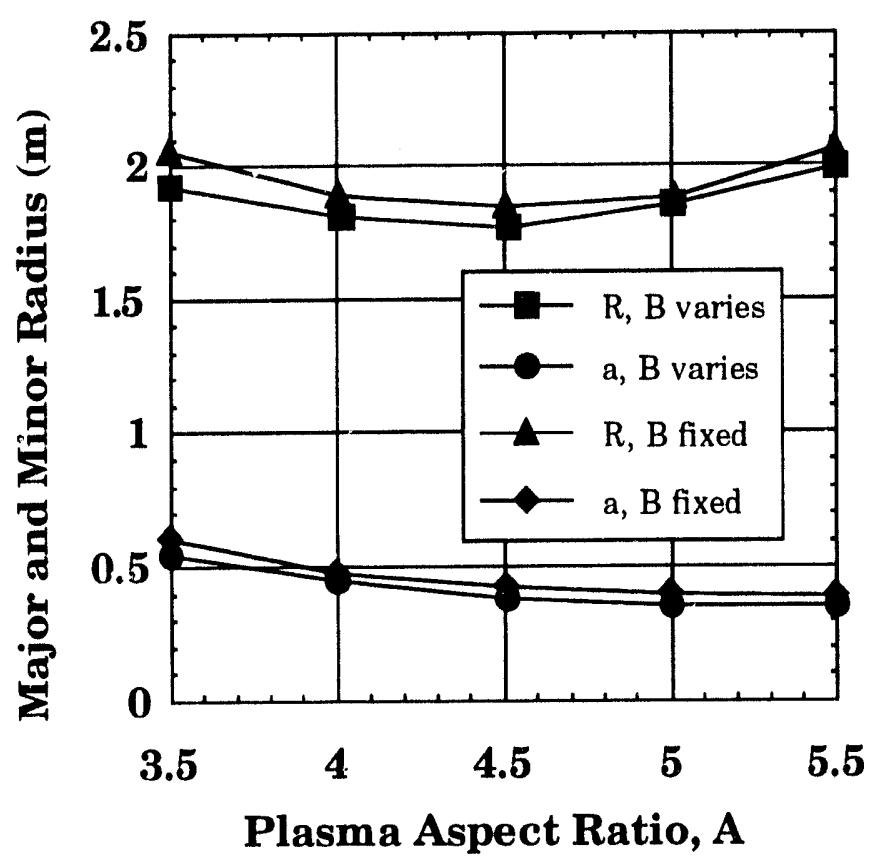

Fig. 4. 'i'PX major toroidal radius, $R_{T}(\mathrm{~m})$, and minor plasma radius, $a(\mathrm{~m})$, as functions of the plasma aspect ratio, $A=R_{T} / a$, assuming fixed (synchronized) or varied on-axis magnetic field, $B_{T}$. The choice $A \simeq 4.5$, near the center of a shallow minimum of $R_{T}$, tends to minimize the size of the device. Use of $B_{T}$ as a fixed variable is not decisive in the choice of these plasma geometrical parameters.

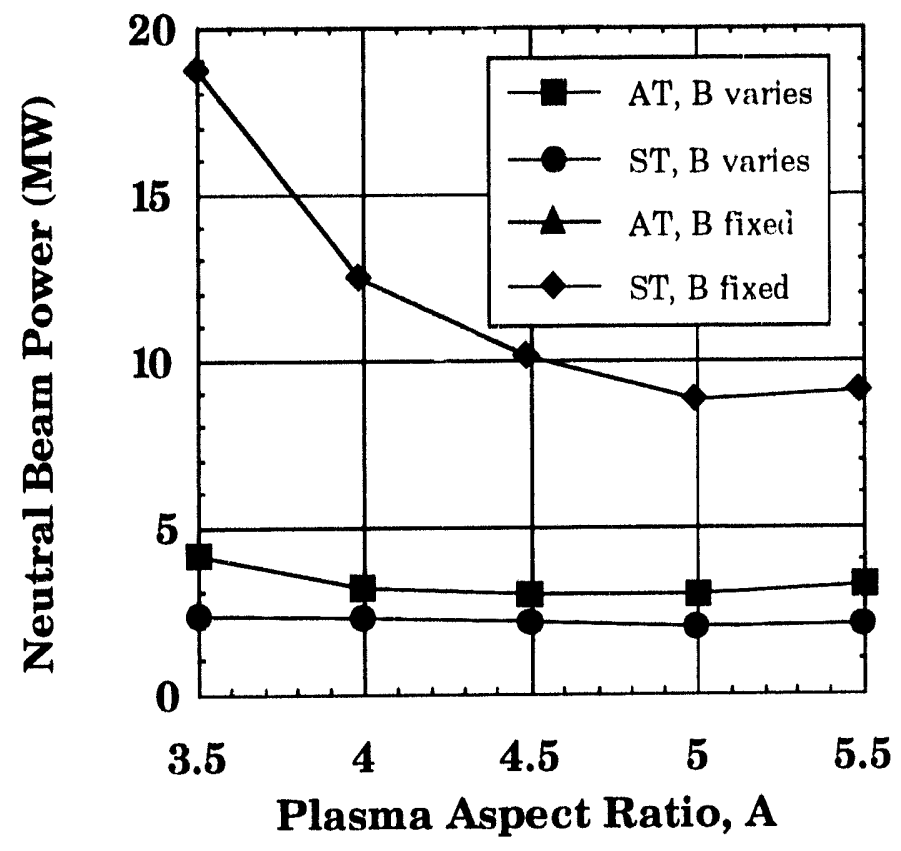

Fig. 5. TPX neutral-beam heating power (MW) as a function of plasma aspect ratio, $A=R_{T} / a$, assuming fixed (synchronized) or varied on-axis magnetic field, $B_{T}$, hetween $\mathrm{ST}^{\prime}$ and AT operating modes. Day-1 constraints on NBI and ICRH power lead to the choice of $A \geq 4.5$. The A'T-mode curves, denoted by squares and triangles, are nearly coincident.

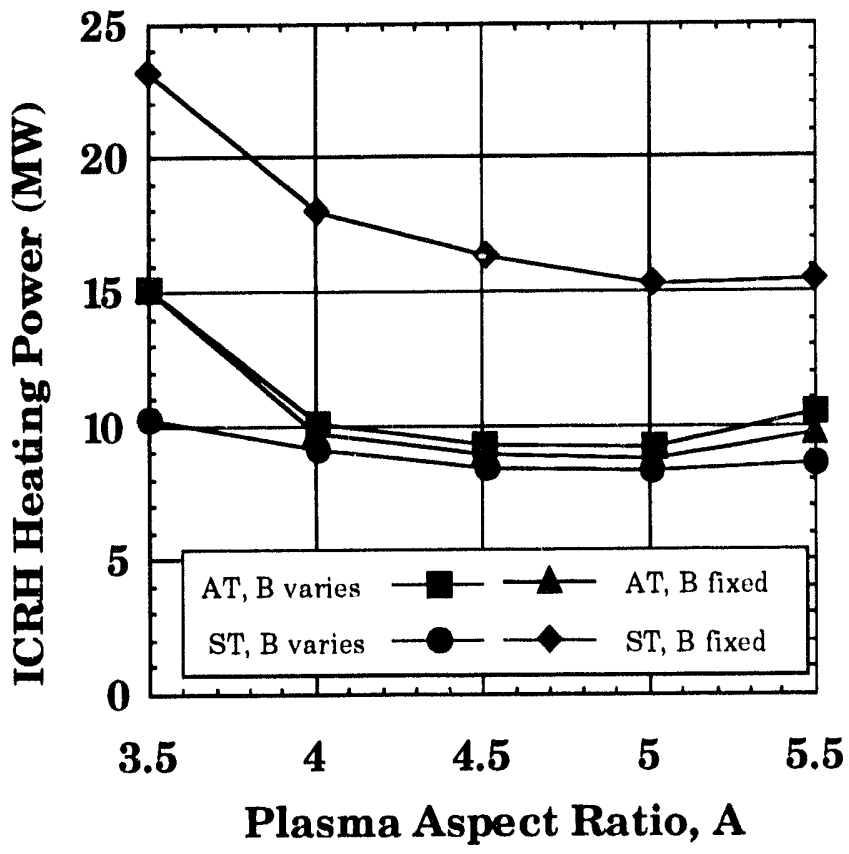

Fig. 6. TPX ICRH heating power (MW) as a function of plasma ratio, $A=R_{T} / a$, assuming fixed (synchronized) or varied on-axis magnetic field, $B_{T}$, between ST and AT operating modes. Day-1 constraints on NBI and ICRH power lead to the choice of $A \simeq 4-5$. 
TABLE I

Summary of TPX Parameters

\begin{tabular}{|c|c|}
\hline PARAMETER & VALUE \\
\hline Plasma aspect ratio, $A=R_{T} / a$ & 4.5 \\
\hline Major toroidal radius, $R_{T}(\mathrm{~m})$ & 2.25 \\
\hline Minor plasma radius, $a(\mathrm{~m})$ & 0.50 \\
\hline Plasma vertical elongation, $\kappa_{x}$ & $1.6-2.0$ \\
\hline Toroidal magnetic field, $B_{T}(\mathrm{~T})$ & 4.0 \\
\hline Plasma current, $I_{p}(\mathrm{MA})$ & 2.0 \\
\hline \multicolumn{2}{|l|}{ Day-1 Heating and Current Drive: } \\
\hline NBI (MW) & 8.0 \\
\hline ICRH (MW) & 8.0 \\
\hline LH $(\mathrm{MW})$ & 1.5 \\
\hline ST-mode $\beta=\beta_{N} I_{p} / a B_{T}$ & 0.035 \\
\hline
\end{tabular}

The cost of new ICRH heating capability dominates the upgrade of existing TFTR NBI units in WBS-2 and the cost of the superconducting Toroidal-Field (TF) coil set dominates WBS-1. Table II summarizes baseline PACE and OPEX costs for TPX according to the higherlevel WBS items. The total device cost in 1992-dollars is $453.1 \mathrm{M} \$$. Due to present space limitations, cost details at the lower WBS levels and the constituents of the PACE and OPEX costs are reported elsewhere [8].

TABLE II

Sample SuPERCODE TPX Cost Summary ${ }^{(a)}$

\begin{tabular}{|c|c|c|}
\hline WBS ITEM & PACE & OPEX \\
\hline 1 Tokamak Systems & 179.4 & 19.2 \\
\hline 11 Plasma Facing Components & 35.0 & 3.8 \\
\hline 12 Vacuum Vessel & 25.4 & 2.2 \\
\hline 13 Toroidal-Field (TF) System & 56.6 & 9.2 \\
\hline 14 Poloidal-Field (PF) System & 26.0 & 2.4 \\
\hline 15 Cryostat & 10.5 & 0.9 \\
\hline 16 Tokamak Support Structure & 2.5 & 0.3 \\
\hline 17 Assembly \& Test & 17.7 & 0 \\
\hline 18 Tokamak Shielding & 5.7 & 0.5 \\
\hline 2 Auxiliary Heating \& CD & 96.2 & 2.1 \\
\hline $21 \mathrm{NBI}$ & 31.0 & 0.9 \\
\hline 23 ICRH & 65.1 & 1.1 \\
\hline $24 \mathrm{LH}$ & 0 & 0 \\
\hline 3 Fueling \& Vacuum Systems & 10.0 & 0.7 \\
\hline 4 Power Systems & 28.0 & 0.5 \\
\hline 5 Maintenance Systems & 16.7 & 8.2 \\
\hline 6 Data Systems & 27.4 & 1.4 \\
\hline 61 Central I\&C & 10.6 & 0.4 \\
\hline 61 Plasma Diagnostics & 16.8 & 1.0 \\
\hline 7 Facilities & 19.5 & 0.2 \\
\hline 8 Preparations for Operations & 0 & 10.4 \\
\hline 9 Project Support & 21.3 & 12.0 \\
\hline Total Device Cost & 398.4 & 54.7 \\
\hline
\end{tabular}

(a) 1992 dollars.

\section{SUMMARY}

The success of TPX improves the fusion-power-plant relevance of the tokamak. Equipped with a detailed cost module, SUPERCODE has been applied to the conceptual design and optimization of the TPX. Key trade and sensitivity studies have been performed, confirming a fairly robust TPX conceptual design at $A \simeq 4.5$. Minimizing projected cost while maximizing performance has been a driving consideration. Operational flexibility is addressed by bi-modal (ST and AT) optimization of device parameters. The demonstrated capability for bi-modal optimization establishes the techniques for future multi-modal optimizations. Use of the SUPERCODE for TPX studies diminishes as the TPX moves into the engineering design phase. The code package developed in this work is being adapted for future studies of demonstration (DEMO) tokamak power plants and other devices.

\section{ACKNOWLEDGEMENT}

The authors acknowledge the assistance of $R . T$. Simmons (PPPL) in the ongoing provision of current TPX cost baselines and useful discussions with R. J. Goldston (PPPL), G. H. Neilson (ORNL), W. M. Nevins (LLNL), and W. T. Reiersen (PPPL).

\section{REFERENCES}

[1] G. H. Neilson et al., "Physics Design Requirements for the Tokamak Physics Experiment (TPX)," these Proceedings.

[2] W. T. Reiersen, "The Engineering Design of TPX," these Proceedings.

[3] G. H. Neilson el al., "Design of a Superconducting Steady State Advanced Tokamak," Proc. 17th Symp. on Fusion Technology, 1992, 2, 1636 (1993).

[4] R. J. Goldston et al., "The Mission and Physics Design of TPX," Princeton Plasma Physics Laboratory report PPPL-CFP-2821 (1993).

[5] F. Najmabadi et al., "The ARIES-II and -IV Second Stability Tokamak Reactors," University of California Los Angles report UCLA-PPG-1461 (to be published).

[6] S. W. Haney et al., "A 'SUPERCODE' for Systems Analysis of Tokamak Experiments and Reactors," Fusion Technology 21, 1749 (May 1992).

[7] R. T. Simmons, "TPX Cost/Schedule Overview," TPX Conceptual Design Review (March 29 - April 1, 1993).

[8] R. L. Miller, "Fusion-Device Costing Module for Use in SUPERCODE," Los Alamos National Laboratory document (to be published, 1993). 

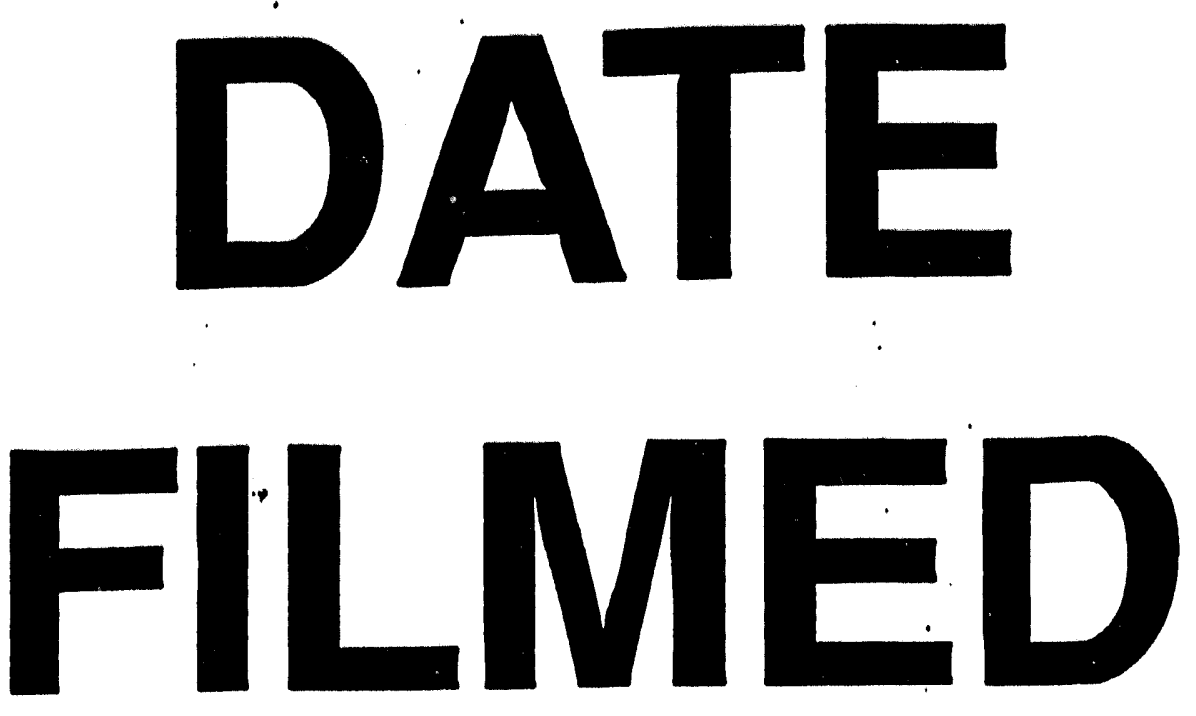

$12 / 27 / 93$
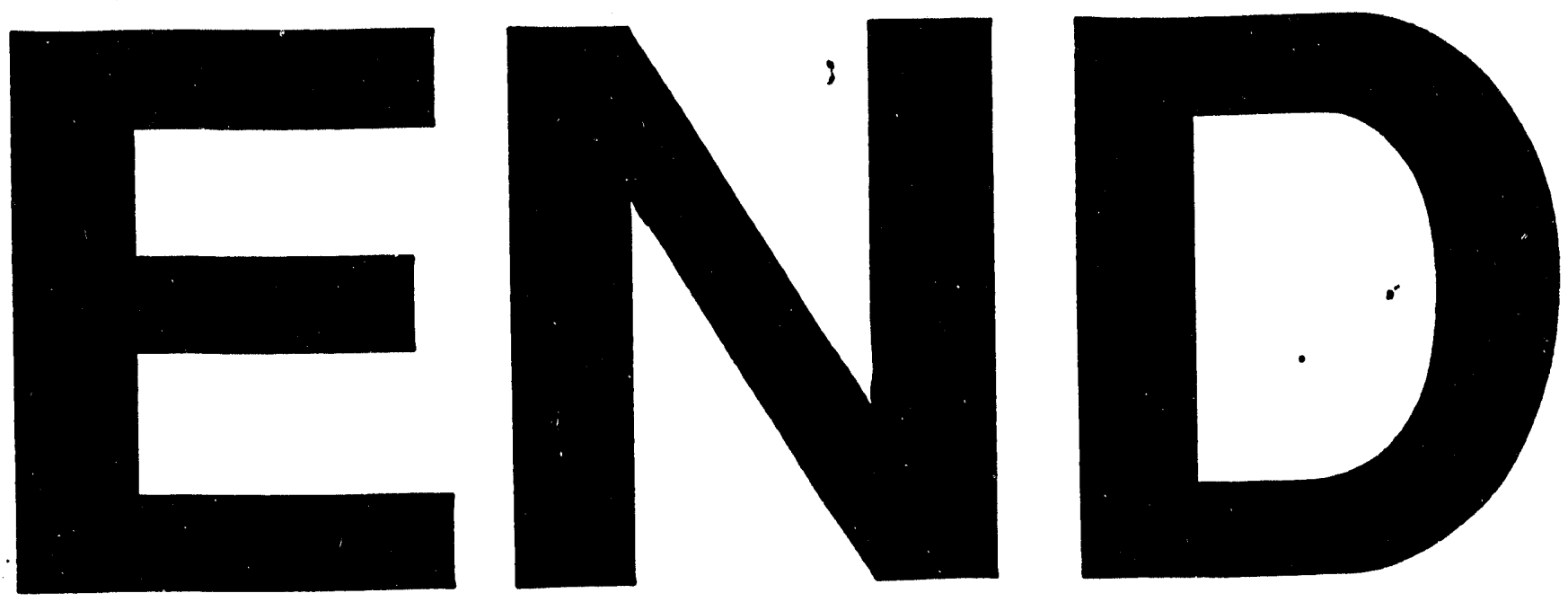
